\title{
Inhibition of peritoneal metastasis of human gastric cancer cells by dextran sulphate through the reduction in HIF-1 $\alpha$ and ITG $\beta 1$ expression
}

\author{
YUANYI XU ${ }^{1 *}$, XIU JIN $^{1 *}$, YUNNING HUANG $^{2 *}$, JIANDA DONG $^{1}$, HONGHONG WANG $^{3}$, \\ XIAOFEI WANG ${ }^{1}$ and XIANGMEI CAO
}

\author{
${ }^{1}$ Department of Pathology, Ningxia Medical University, Yinchuan, Ningxia 750001; Departments of ${ }^{2}$ Gastrointestinal Surgery \\ and ${ }^{3}$ Pathology, Ningxia People's Hospital, Yinchuan, Ningxia 750021, P.R. China
}

Received November 26, 2015; Accepted December 31, 2015

DOI: $10.3892 /$ or.2016.4693

\begin{abstract}
The aim of the present study was to investigate the effects of dextran sulphate (DS) on HIF-1 $\alpha$ and integrin $\beta 1$ (ITG $\beta 1$ ) expression in human gastric cancer cells, the correlation between HIF- $1 \alpha$ and ITG $\beta 1$ expression and the influence of DS on the peritoneal metastasis of human gastric cancer cells. In in vitro experiments, BGC-823 cells in the experimental and control groups were administered DS and PBS, respectively, and exposed to hypoxic conditions for different periods. Immunocytochemistry, western blot and RT-PCR analyses were used to evaluate HIF- $1 \alpha$ and ITG $\beta 1$ expression levels. In in vivo experiments, an animal model was established by injecting BGC-823 cells into nude mice. The experimental and control groups received DS and PBS injections, respectively. The mice were euthanized at different times, and the number of tumor nodules in the celiac implantation was recorded. Immunohistochemistry, RT-PCR and western blot analyses were used to detect HIF-1 $\alpha$ and ITG $\beta 1$ expression in the tumor nodules of the greater omentum. The in vitro and in vivo results revealed that HIF- $1 \alpha$ and ITG $\beta 1$ expression levels in the experimental group were significantly lower than those in the control group $(\mathrm{P}<0.05)$, and the expression levels of these factors were positively correlated with each
\end{abstract}

Correspondence to: Dr Yuanyi Xu, Department of Pathology, Ningxia Medical University, Yinchuan, 1160 Shengli Street, Xingqing, Yinchuan, Ningxia 750001, P.R. China

E-mail:nxxyy@hotmail.com

Dr Yunning Huang, Department of Gastrointestinal Surgery, Ningxia People's Hospital, 301 Zhengyuan North Street, Jinfeng, Yinchuan, Ningxia 750021, P.R. China

E-mail: nxhyncc@126.com

${ }^{*}$ Contributed equally

Key words: dextran sulphate, human gastric cancer cells, hypoxia-inducible factor- $1 \alpha$, integrin $\beta 1$, peritoneal metastasis other. The number of tumor nodules in the in vivo experiments was notably less in the experimental group than that noted in the control group $(\mathrm{P}<0.01)$. In conclusion, DS may act through inhibition of HIF-1 $\alpha$ expression, which decreased ITG $\beta 1$ expression, consequently reducing tumor metastasis.

\section{Introduction}

Gastric cancer is one of the most common types of gastrointestinal tumors; its incidence and mortality are among the highest for all types of malignant tumors. The metastatic implantation of gastric cancer within the abdominal cavity is the leading cause of death in patients with gastric cancer, and effective approaches for its prevention and treatment are critically needed. The primary process of abdominal implantation of gastric cancer consists of cancer cell infiltration into the serous layer of the stomach, detachment into the abdominal cavity, and formation of an active tumor mass that implants and proliferates to form metastases (1).

Cell mobility is an important characteristic that allows tumor cells to invade surrounding tissue and form distant metastases. Hypoxia can increase tumor cell mobility and migration, which are closely related to tumor metastasis (2). In a hypoxic microenvironment, hypoxia-inducible factor- $1 \alpha$ (HIF-1 $\alpha)$ is highly expressed in tumor cells (3). HIF-1 $\alpha$ is a specific transcription factor that plays an active role under hypoxic conditions. High expression of HIF-1 $\alpha$ can promote epithelial-mesenchymal transition (EMT), regulate angiogenesis (via VEGF), and increase cell adhesion [via integrin $\beta 1$ (ITG $\beta 1$ )] to allow cells to adapt to a hypoxic microenvironment consequently promoting tumor growth and metastasis $(4,5)$.

As a key regulator of the hypoxia response, HIF-1 $\alpha$ promotes the expression of ITG $\beta 1$ to increase the adhesion of induced cells (5). Adhesion is important to the process of gastric cancer metastasis. Integrin is a member of the adhesion factor receptor family. ITG $\beta 1$, an important $\beta$ subunit of the integrin family, can combine with different $\alpha$ subunits and form the vast majority of extracellular matrix receptors. ITG $\beta 1$ plays a mediating role in cell-cell and cell-cell matrix interactions (6). Enhanced ITG $\beta 1$ expression is related to celiac implantation of cancer (7). Therefore, studies aimed at 
examining HIF-1 $\alpha$ and ITG $\beta 1$ expression are important for the treatment of tumor recurrence and metastasis.

Animal models have shown that dextran sulphate (DS) can prevent B-16 melanoma cells from implanting in great omentum milky spots and the peritoneum and can prolong the survival of mice with cancerous peritonitis (8). We hypothesized that DS may inhibit peritoneal metastasis of human gastric cancer cells through a reduction in HIF-1 $\alpha$ and ITGB1 expression. In the present study, gastric cancer cells were exposed to DS in vitro, and the expression levels of HIF-1 $\alpha$ and ITG $\beta 1$ were measured and subjected to correlation analysis. Furthermore, gastric cancer cells were injected into nude mice to create a model of gastric carcinoma peritoneal metastasis in vivo and to observe the influence of DS on the nodules on the greater omentum. HIF- $1 \alpha$ and ITG $\beta 1$ expression in the greater omentum was evaluated for further validation of the in vitro experiment. Through in vivo and in vitro experiments, the effects of DS on celiac metastasis of human gastric cancer cells were evaluated.

\section{Materials and methods}

\section{Materials}

Cell culture. The human gastric cancer cell line BGC-823 (Beijing Jin Zijing Biological Pharmaceutical Technology Co. Ltd., Tumor Cell Library) was cultured in RPMI-1640 medium (Hyclone), fetal bovine serum (FBS) (Fumeng Gene), trypsinEDTA (Solarbio), and streptomycin.

Drugs. DS (Sigma) was dissolved in phosphate-buffered saline (PBS) for cell culture or in $0.9 \%$ normal saline for injection into nude mice and then sterilized with a $22-\mu \mathrm{m}$ filter; the final concentration was $0.3 \%$.

Experimental animals. BALB/c nude mice were purchased from Beijing Tong Lihua Experimental Animal Technical Co., Ltd. (animal license SCXX, Beijing, 2006-0009); the animals were 5 to 6 weeks old, male, and 18 to $22 \mathrm{~g}$ in weight. All procedures were performed according to the animal protocol approved by the Ningxia Medical University Laboratory Animal Centre and internationally recognized guidelines on animal welfare. Aseptic food and water were available ad libitum.

Antibodies and reagents. The following antibodies were used: ITG $\beta 1$ polyclonal antibody (Proteintech Group), sheep monoclonal antibody against ITG $\beta 1$, rabbit anti-sheep $\mathrm{IgG}$, goat anti-rabbit IgG and HIF-1 $\alpha$ polyclonal antibody (Proteintech Biological Co., Ltd.) and sheep anti-rabbit $\beta$-actin (Elabscience). The western blot reagents were as follows: a total protein extraction kit (KeyGen), a protein quantitative kit (Kang Century), and an enhanced chemiluminescence kit HRP (Fdbio Science). The PCR reagents were as follows: a total mRNA extraction kit (Omega), Revert Aid First Strand cDNA Synthesis kit (Thermo) and PCR kits (Beijing CoWin Biotech Co., Ltd.). The immunohistochemical reagents used were as follows: goat anti-rabbit IgG and DAB kit (both from ZSGB-Bio).

\section{Methods for the in vitro experiments}

Cell culture. BGC-823 cells were maintained under routine culture conditions in complete medium in a sterile incubator at a constant temperature of $37^{\circ} \mathrm{C}$ with $5 \% \mathrm{CO}_{2}$. The medium was replaced two or three times per week, and the cells were passaged every three days. The cells were grown to the logarithmic phase in a $60-\mathrm{mm}$ Petri dish. The control and experimental groups received PBS and DS (final concentration of $0.3 \%$ ), respectively, and the cells were placed in a low-oxygen incubator at $37^{\circ} \mathrm{C}$ with $5 \% \mathrm{CO}_{2}$ and $1 \% \mathrm{O}_{2}$. The cells were collected after 2, 8, 12 and $24 \mathrm{~h}$ for evaluation.

Immunocytochemical staining. The treated cells were fixed in cold 4\% polyphosphate formaldehyde for $15 \mathrm{~min}$ and then washed with PBS. The fixed cells were permeabilized with $0.5 \%$ Triton $\mathrm{X}-100$ for $30 \mathrm{~min}$ and subsequently blocked with goat serum for $30 \mathrm{~min}$ at $37^{\circ} \mathrm{C}$. After the cells were washed in PBS, they were incubated with anti-HIF-1 $\alpha$ (1:100) or anti-ITG $\beta 1$ (1:100) at $4^{\circ} \mathrm{C}$ overnight, followed by incubation with goat anti-rabbit secondary antibodies for $1 \mathrm{~h}$ at room temperature. DAB staining was performed for 3-5 min before cell evaluation under a microscope. Finally, the cells were counterstained with haematoxylin. Positive HIF-1 $\alpha$ staining was observed in the nucleus and cytoplasm and was assessed using Image-Pro Plus 6.0 software (IPP) to obtain the average optical density (OD) value of the selected field.

Measurement of HIF-1 $\alpha$ and ITG $\beta 1 \mathrm{mRNA}$ levels by $R T-P C R$. Cells that were treated for different periods were lysed, and total RNA was extracted and reverse transcribed using a total mRNA extraction kit and a reverse transcription kit according to the manufacturer's instructions. The primers for HIF- $1 \alpha$ and ITG $\beta 1$ were designed and purchased from Shanghai Sangon Biological Engineering Co., Ltd., and were as follows: ITG $\beta 1$ forward, 5'-CGTAGCAAAGGAACAGC AGA-3' and reverse, 5'-GTAAGACAGGTCCATAAGGTAG TAG-3', and HIF-1 $\alpha$ forward, 5'-GAAAGCGCAAGTCTTCA AAG-3' and reverse, 5'-TGGGTAGGAGATGGAGATGC-3'. The RT-PCR assay was analyzed by grey value analysis using Quantity One software; the target/internal relative grey-scale value indicates the corresponding mRNA expression level. The ITG $\beta 1$ and HIF-1 $\alpha$ mRNA expression levels in cells were compared between the experimental and control groups at several time-points.

Western blot detection of HIF-1 $\alpha$ and ITG $\beta 1$ protein levels. The cells treated with PBS or DS were harvested, lysed, subjected to SDS-polyacrylamide gel electrophoresis and transferred to polyvinylidene fluoride (PVDF) membranes. The membranes were blocked with $10 \%$ skim milk for $1.5 \mathrm{~h}$ at room temperature, and the immunoreactivity levels were evaluated by hybridization with the following antibodies: rabbit polyclonal anti-HIF-1 $\alpha$ (1:1,000), anti-ITG $\beta 1$ (1:500) and anti- $\beta$-actin $(1: 1,000)$ antibodies and goat anti-rabbit IgG $(1: 6,000)$. The signal was then detected by chemiluminescence using an ECL kit and exposure in a darkroom or an Amersham Imager 600 instrument. For grey value analysis with Quantity One, the target/internal relative grey scale value indicates the corresponding protein expression level. HIF-1 $\alpha$ and ITG $\beta 1$ expression levels in cells were compared between the experimental and control groups at several time-points.

\section{Methods for the in vivo experiments}

Establishment of a nude mouse model of gastric celiac metastasis. Ninety $\mathrm{BALB} / \mathrm{c}$ nude mice were randomly divided into a control group $(\mathrm{n}=40)$ and an experimental group $(n=50)$. Experimental procedures were performed with 
approval from the Animal Care Committee of our institute. The nude mice were anaesthetized with $0.5 \%$ pentobarbital sodium and the stomach walls were exposed. BGC-823 cells (2x10\% $/ 0.2 \mathrm{ml} /$ mouse) and $0.3 \% \mathrm{DS}(1 \mathrm{ml} /$ mouse) suspended in PBS were injected subcutaneously into the stomach area of the nude mice in the experimental group. Under the same conditions, the mice in the control group were injected with BGC-823 cells suspended in PBS and $0.9 \%$ saline. The control group and experimental group were randomly divided into four groups each.

Mice were euthanized at different time-points. Each group of nude mice injected with drugs and tumor cells was sacrificed by cervical dislocation on day 1, 2, 3, 7 or 14; a natural death group was also established. The number, size and color of tumor nodules in the abdominal cavity, particularly on the greater omentum, were observed. Two samples of implanted tumor nodules were collected from each mouse: one sample was immediately placed in $10 \%$ formalin $(\mathrm{pH}$ 7.4) for $\mathrm{H} \& \mathrm{E}$ and immunohistochemical staining, and the other was cryopreserved at $-80^{\circ} \mathrm{C}$ in a cryogenic vial, which had been disinfected and treated with DEPC water, for later evaluation.

$H \& E$ and immunohistochemical staining. Formalin-fixed and paraffin-embedded specimens were sectioned and fixed in $10 \%$ formalin for up to $24 \mathrm{~h}$. The slices were then deparaffinized, the antigen was retrieved, and the samples were incubated in hydrogen peroxide for $15 \mathrm{~min}$. Primary antibody was added to each slice and incubated at $4^{\circ} \mathrm{C}$ overnight; PBS was used as a negative control. HRP polymer (HRP secondary antibody) was added to the washed slides, and the slides were incubated at $37^{\circ} \mathrm{C}$ for $30 \mathrm{~min}$. Then, $100 \mu \mathrm{l}$ of DAB working solution was added drop-wise to each slide, and the slides were incubated for 3-10 min. The slides were then washed, counterstained and dehydrated before the transparent mounting medium and coverslips were added. The IPP image automatic analysis system was used at $x 400$ to select a blank area and five representative areas. The OD value of each area was determined and calculated, and the average OD value was obtained for the selected field .

RT-PCR detection of HIF-1 $\alpha$ and ITG 1 mRNA expression in the greater omentum. Total RNA was extracted from tissue using a total mRNA extraction kit. RNA was then reverse transcribed using a reverse transcription kit according to the manufacturer's instructions. The primers used for RT-PCR were as follows: ITG $\beta 1$ forward, 5'-CGTAGCAAAGGAAC AGCAGA-3' and reverse, 5'-GTAAGACAGGTCCATAAGG TAGTAG-3'; and HIF-1 $\alpha$ forward, 5'-GAAAGCGCAAGTCT TCAAAG-3' and reverse, 5'-TGGGTAGGAGATGGAGA TGC-3'. This process was followed by grey value analysis with Quantity One; the target/internal relative grey-scale value indicated the corresponding mRNA expression level. The ITG $\beta 1$ and HIF-1 $\alpha$ mRNA expression levels were compared between the experimental and control groups at several time points.

Western blot detection of HIF-1 $\alpha$ and ITG $\beta 1$ protein expression. Tumor tissues were sheared and added to pyrolysis liquid. The slurry was centrifuged at $14,000 \mathrm{rpm}$ at $4^{\circ} \mathrm{C}$ for $15 \mathrm{~min}$. The supernatant was used to extract the total protein, and the BCA method was used to quantify the protein levels. The proteins were subjected to SDS-polyacrylamide gel electrophoresis, transferred to PVDF membranes, and blocked for $1.5 \mathrm{~h}$. Immunoreactivity levels were evaluated by hybridization using the following antibodies: rabbit polyclonal anti-HIF- $1 \alpha$ and anti-ITG $\beta 1$ antibodies and goat anti-rabbit IgG. The signal was then detected by chemiluminescence using an ECL kit and exposure in a darkroom or an Amersham Imager 600 instrument. Grey value analysis was performed with Quantity One to compare HIF- $1 \alpha$ and ITG $\beta 1$ expression levels between the experimental and control groups at different time-points.

Statistical analyses. The data were analyzed using SPSS 17.0 statistical software. The comparison of two sample means was performed using the two-sample t-test. Multiple samples were compared using analysis of variance and correlations between the two factors were analyzed using Pearson's correlation analysis.

\section{Results}

\section{In vitro experimental results}

Immunocytochemical staining. HIF- $1 \alpha$ was expressed in the nucleus and cytoplasm of the control and experimental BGC-823 cell groups; tan color indicates positive expression. After exposure to hypoxia for $2(\mathrm{P}<0.05), 8(\mathrm{P}<0.01), 12$ $(\mathrm{P}<0.01)$ or $24 \mathrm{~h}(\mathrm{P}<0.01)$, the level of positive expression in the experimental group was significantly decreased compared with that in the control group (Fig. 1A). IGT $\beta 1$ was expressed in the cell membrane and the cytoplasm; tan colour indicates positive expression. After exposure to hypoxia for $2 \mathrm{~h}$, positive expression in the experimental group was slightly decreased compared with that in the control group; however, no significant difference was observed ( $\mathrm{P}>0.05)$. After exposure to hypoxia for $8(\mathrm{P}<0.01), 12(\mathrm{P}<0.01)$, or $24 \mathrm{~h}(\mathrm{P}<0.01)$, the rate of positive expression was significantly decreased in the experimental group compared with that noted in the control group (Fig. 1B).

$R T$-PCR detection of HIF-1 $\alpha$ and ITG 1 I mRNA expression in the gastric cancer cells. HIF- $1 \alpha$ mRNA expression was significantly lower in the experimental group than that noted in the control group at each time point $(2,8,12$, and $24 \mathrm{~h})$. ITG $\beta 1 \mathrm{mRNA}$ expression did not differ significantly between the two groups at $2 \mathrm{~h}$. However, at 8,12 and $24 \mathrm{~h}$, the expression levels were significantly lower in the experimental group than levels in the control group (Fig. 2).

Western blot detection of HIF-I $\alpha$ and ITG $\beta 1$ protein expression in gastric cancer cells. HIF-1 $\alpha$ protein expression was significantly lower in the experimental group than that noted in the control group at all time-points $(2,8,12$ and $24 \mathrm{~h})$. ITG $\beta 1$ protein expression was reduced in the experimental group compared with that in the control group at each timepoint; no significant difference between groups was observed at $2 \mathrm{~h}$, but the differences were significant at 8,12 , and $24 \mathrm{~h}$ (Fig. 3).

Correlation analysis of HIF-1 $\alpha$ and ITG $\beta 1$ protein expression and time in hypoxia. Pearson's correlation analysis of the HIF-1 $\alpha$ and ITG $\beta 1$ protein target/internal grey values and hypoxia time indicated that ITG $\beta 1$ and HIF- $1 \alpha$ protein expression levels were positively correlated with each other and that the level of each was positively correlated with the time in hypoxia (Table I and Fig. 3). 


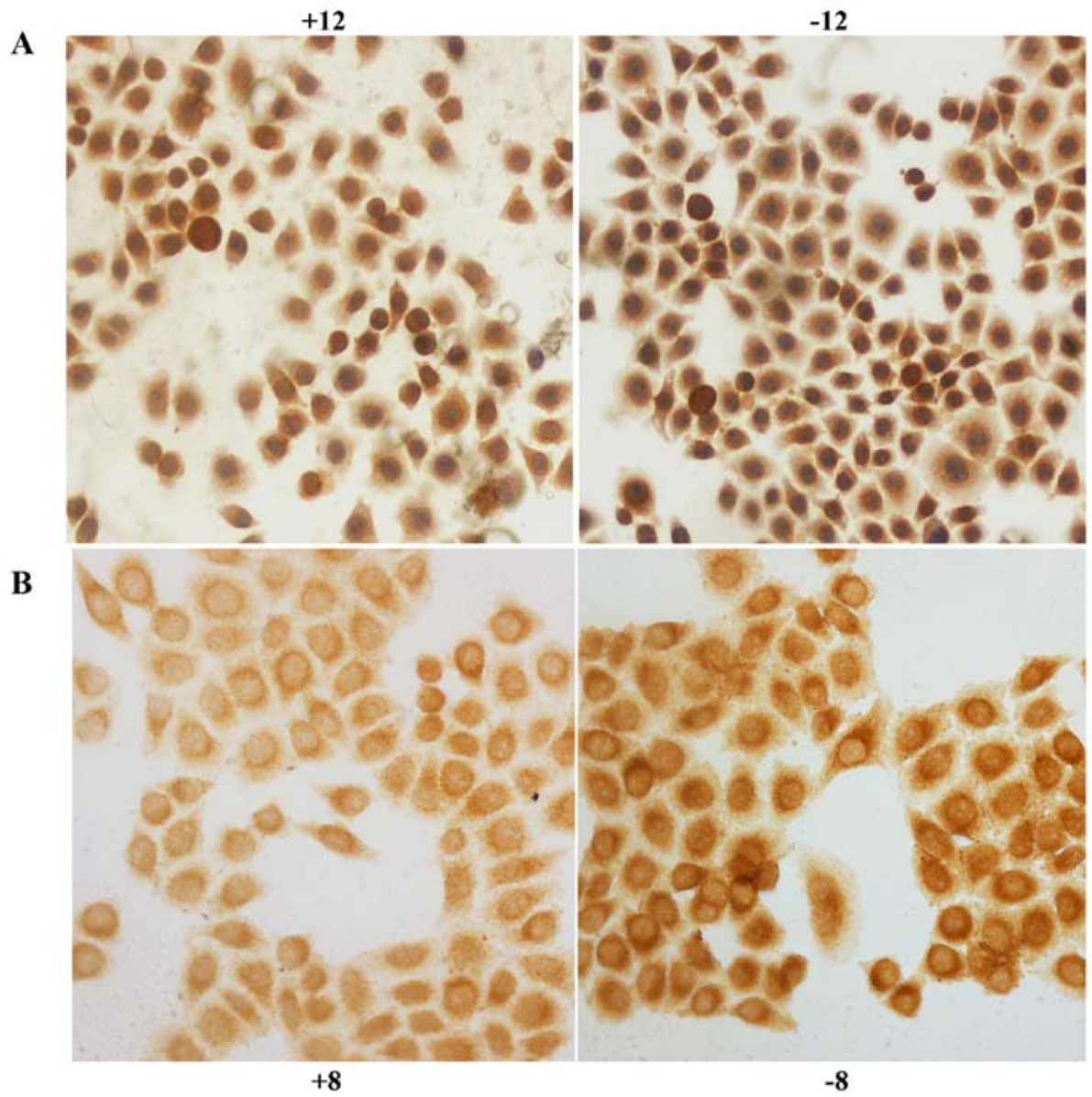

Figure 1. (A) HIF-1 $\alpha$ immunocytochemical staining in the BGC- 823 control and experimental cell groups at $12 \mathrm{~h}$; the labels +12 and -12 indicate the experimental group and control group, respectively, at $12 \mathrm{~h}$. (B) ITG $\beta 1$ immunocytochemical staining in the control and experimental groups at $8 \mathrm{~h}$; the labels +8 and -8 indicate the experimental group and control group, respectively, at $8 \mathrm{~h}$.

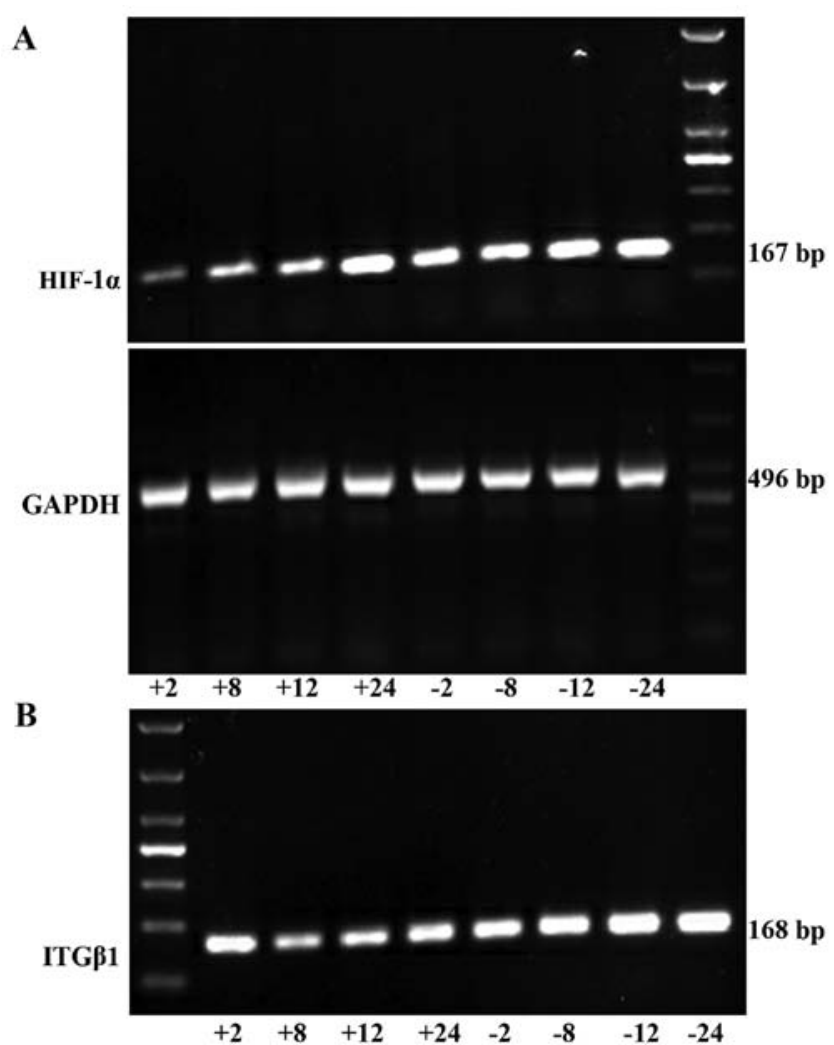

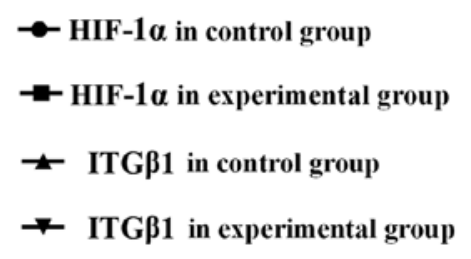

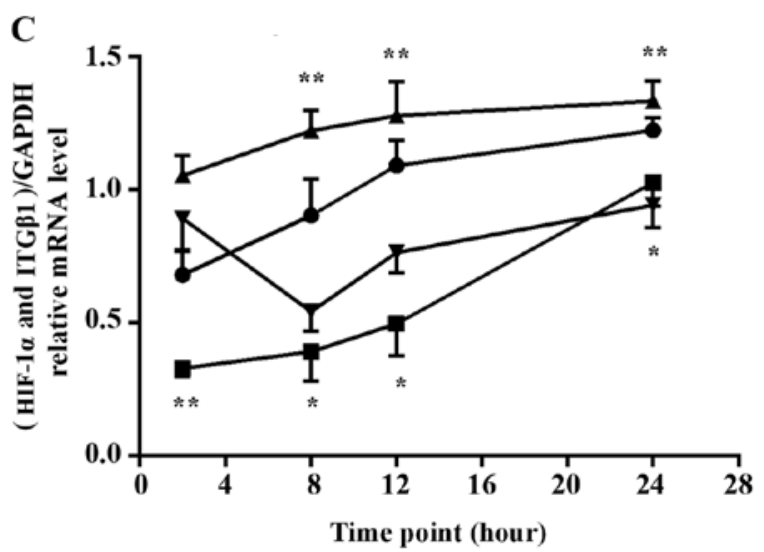

Figure 2. RT-PCR was used to detect HIF-1 $\alpha$ (A) and ITG $\beta 1$ (B) mRNA expression levels in the experimental group and the control group in vitro. (C) Line graph of the gray values of the relative HIF-1 $\alpha$ and ITG $\beta 1$ mRNA expression. The labels $+2,+8,+12,+24$, indicate the experimental group at $2,8,12$, and $24 \mathrm{~h}$, respectively, and the labels $-2,-8,-12$, and -24 indicate the control group at $2,8,12$, and $24 \mathrm{~h}$, respectively. ${ }^{*} \mathrm{P}<0.05$ or ${ }^{* *} \mathrm{P}<0.01$, difference between the experimental group and the control group. 

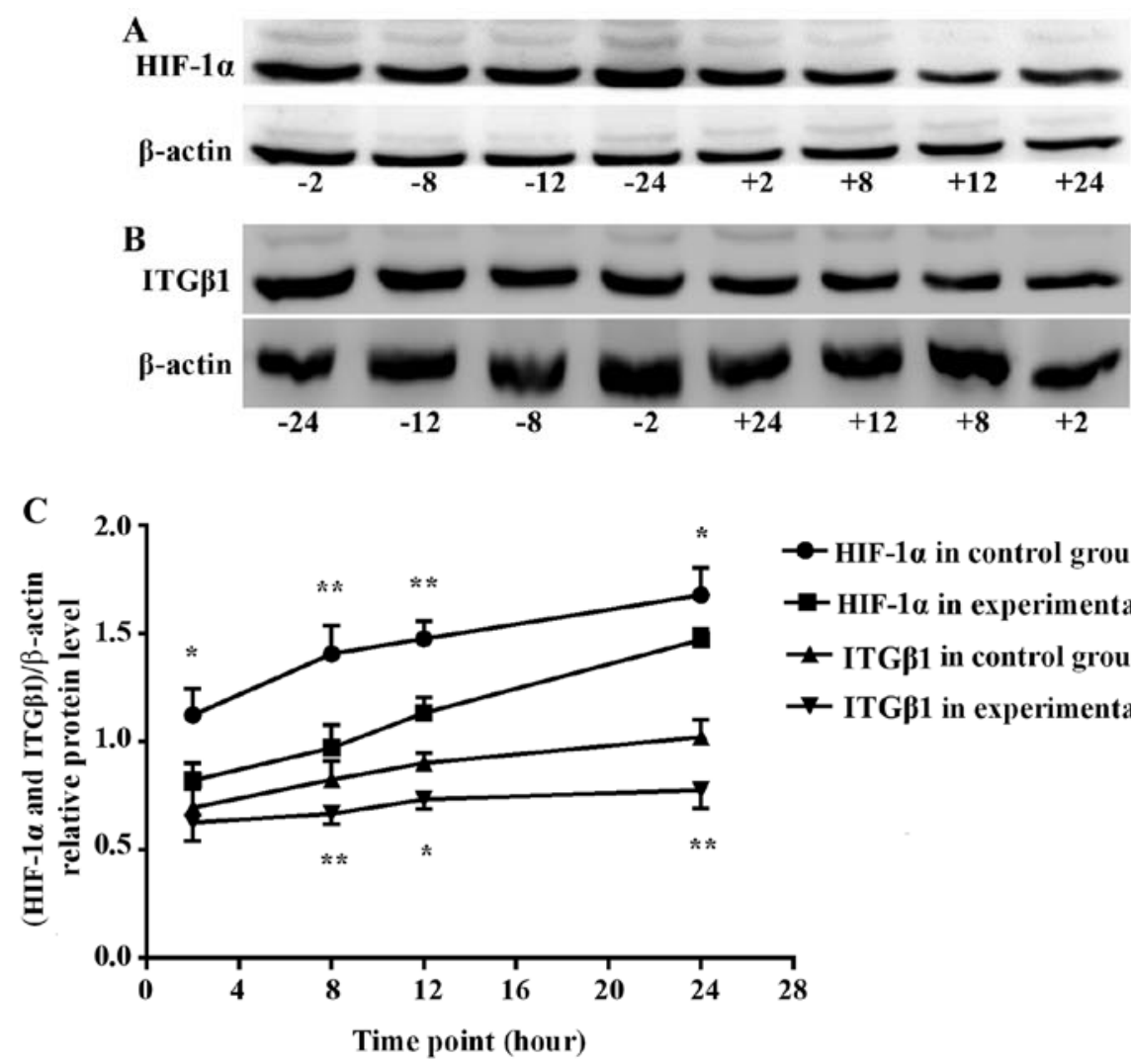

Figure 3. Western blot analysis of HIF-1 $\alpha$ (A) and ITG $\beta 1$ (B) protein expression levels in the BGC-823 experimental and the control cell groups. (C) Line graph of the gray values of the relative HIF- $1 \alpha$ and ITG $\beta 1$ protein expression. The labels $+2,+8,+12,+24$, indicate the experimental group at $2,8,12$, and $24 \mathrm{~h}$, respectively, and the labels $-2,-8,-12$, and -24 indicate the control group at $2,8,12$, and 24 h, respectively. ${ }^{*} \mathrm{P}<0.05$ or ${ }^{* *} \mathrm{P}<0.01$, difference between the experimental group and the control group.

Table I. Correlations of HIF- $1 \alpha$ and ITG $\beta 1$ protein expression levels and time in hypoxia.

\begin{tabular}{|c|c|c|c|c|}
\hline & & Time in hypoxia & HIF- $1 \alpha$ & ITG $\beta 1$ \\
\hline \multirow[t]{3}{*}{ Hypoxia time } & Pearson's correlation & 1 & $0.789^{\mathrm{a}}$ & $0.753^{\mathrm{a}}$ \\
\hline & Significance (two-sided) & & 0.020 & 0.031 \\
\hline & $\mathrm{N}$ & 8 & 8 & 8 \\
\hline \multirow[t]{3}{*}{ HIF- $1 \alpha$} & Pearson's correlation & $0.789^{\mathrm{a}}$ & 1 & $0.906^{\mathrm{b}}$ \\
\hline & Significance (two-sided) & 0.020 & & 0.002 \\
\hline & $\mathrm{N}$ & 8 & 8 & 8 \\
\hline \multirow[t]{3}{*}{ ITG $\beta 1$} & Pearson's correlation & $0.753^{\mathrm{a}}$ & $0.906^{\mathrm{b}}$ & 1 \\
\hline & Significance (two-sided) & 0.031 & 0.002 & \\
\hline & $\mathrm{N}$ & 8 & 8 & 8 \\
\hline
\end{tabular}

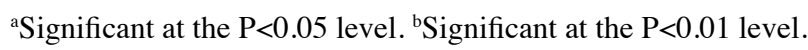

\section{In vivo results}

The number of tumor nodules in the abdominal cavities of mice in the experimental and control groups. After the injection of drugs and cells, the numbers of tumor nodules on the greater omentum, abdominal wall and mesentery gradually increased over time in the experimental and control groups. The nodules appeared on the greater omentum first and were the most numerous. The tumor nodules on the greater omentum were unequal in size and pale with a slightly hard texture and a smooth surface. Some nodules fused and remained independent but lacked well-defined borders and well-defined notes for independent nodules. On the third day, the tumor nodules in the experimental group and control group showed no obvious differences $(\mathrm{P}>0.05)$. At 7 days $(\mathrm{P}=0.003)$ and 14 days $(\mathrm{P}=0.000)$, the nodule number and diameter in the experimental group were significantly reduced compared with those of the control group (Fig. 4).

Immunohistochemical detection of the expression of $H I F-1 \alpha$ and ITG $\beta 1$ in the experimental and control groups at different times. HIF-1 $\alpha$ was expressed in the nucleus and 

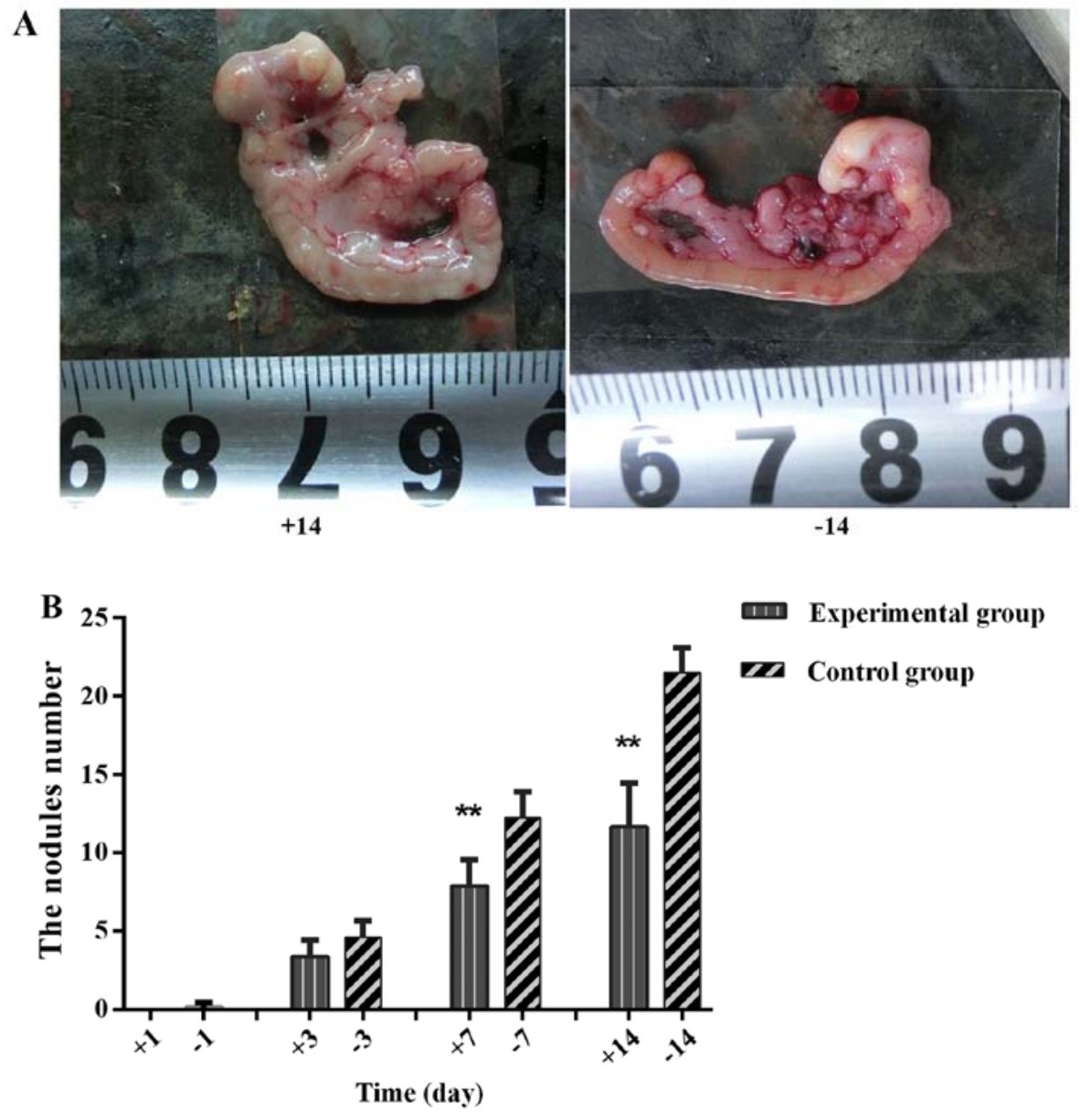

Figure 4. Comparison of abdominal tumor nodules between the experimental and control groups. (A) Images of the greater omentum in mice on day 14. (B) Histogram of the numbers of nodules on the greater omentum. The labels $+1,+3,+7$, and +14 indicate day $1,3,7$ and 14 of the experimental group, respectively, and the labels $-1,-3,-7$, and -14 indicate day 1,3,7 and 14 of the control group, respectively. The numbers of tumor nodules at day 7 and 14 were significantly reduced in the experimental group compared with the controls. ${ }^{* *} \mathrm{P}<0.01$, difference between the experimental group and the control group.

cytoplasm. On day 1 , no obvious difference was found between the experimental group and the control group $(\mathrm{P}>0.05)$. On days 3,7 and 14, expression was significantly reduced in the experimental group compared with that noted in the control group ( $\mathrm{P}<0.01$, Fig. 5A and Table II). ITG $\beta 1$ was expressed in the cytoplasm, and its expression was significantly lower in the experimental group than that in the control group $(\mathrm{P}<0.05$, Fig. 5B and Table III).

RT-PCR detection of HIF-1 $\alpha$ and ITG $\beta 1$ expression on the greater omentum. Analysis of the grey values with image analysis software showed that the expression levels of HIF-1 $\alpha$ and ITG $\beta 1$ in the experimental group were significantly lower than those in the control group at each time point (Fig. 6 and Tables IV and V).

Western blot detection of expression of HIF-1 $\alpha$ and ITG $\beta 1$ in tumor nodules. Analysis of the grey values with image analysis software showed that the expression levels of HIF-1 $\alpha$ and ITG $\beta 1$ in the experimental group were significantly lower than those in the control group at days 3, 7 and 14 (Fig. 7).

Correlation analysis of tumor nodule number, HIF-l $\alpha$ and ITG $\beta 1$ protein expression levels and time since injection. In the experimental and control groups, tumor nodule number on the greater omentum, HIF-1 $\alpha$ and ITG $\beta 1$ target/internal grey values, and drug and cell injection time were subjected to Pearson's correlation analysis. Nodule number and HIF-1 $\alpha$ and ITG $\beta 1$ protein expression levels were all positively correlated with the time since injection. HIF- $1 \alpha$ and ITG $\beta 1$ protein expression levels were positively correlated with tumor nodule number, and HIF- $1 \alpha$ and ITG $\beta 1$ protein expression levels were positively correlated with each other (Table VI and Fig. 7).

\section{Discussion}

Gastric cancer is one of the most common gastrointestinal malignant tumors in China. The morbidity and mortality of gastric cancer are among the highest for all types of malignant tumors, and recurrence and metastasis are the primary causes of the high mortality rate of gastric cancer. Currently, more than half of patients with gastric cancer eventually die of peritoneal implantation. Therefore, methods for preventing gastric cancer and treating it after its spread to the abdominal cavity are urgently needed. The primary process of abdominal implantation of gastric cancer consists of cancer cell infiltration into the serous layer of the stomach, detachment into the abdominal cavity, and formation of an active tumor mass that implants and proliferates to form metastases (1).

After gastric cancer cells disperse into the abdominal cavity, they are likely to grow in the intra-abdominal viscera 

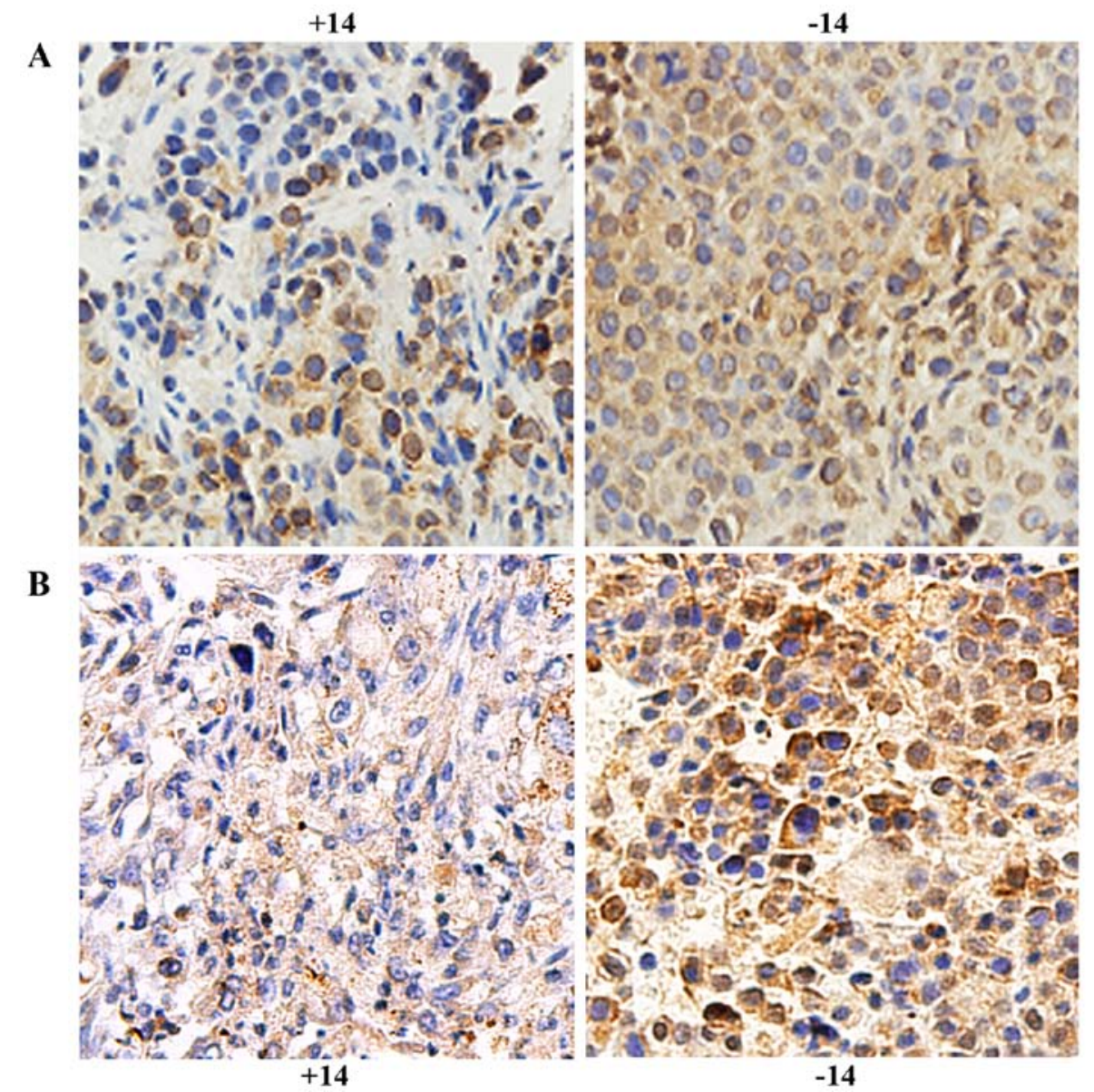

Figure 5. Immunohistochemical detection of HIF-1 $\alpha$ (A) and ITG $\beta 1$ (B) in in stomach cancer tissue of the experimental and control groups at day 14 . The labels +14 and -14 indicate the experimental group and the control group on day 14, respectively. Tan color indicates positive expression.

Table II. The average optical density values of the immunohistochemical staining for HIF-1 $\alpha$ expression in the control and experimental groups at different time-points.

\begin{tabular}{lcccccr}
\hline Group & Day 1 & Day 3 & Day 7 & Day 14 & F & P-value \\
\hline Control & $57.87 \pm 4.36$ & $75.27 \pm 3.36$ & $95.41 \pm 4.81$ & $138.0 \pm 4.30$ & 328.88 & $<0.001$ \\
Experimental & $54.39 \pm 2.18$ & $68.04 \pm 3.25$ & $83.54 \pm 3.77$ & $88.21 \pm 4.51$ & 96.195 & $<0.001$ \\
$t$ & 1.577 & 3.456 & 4.338 & 17.881 & & \\
P-value & 0.154 & 0.009 & 0.002 & $<0.001$ & & \\
\hline
\end{tabular}

Table III. Immunohistochemical staining for ITG $\beta 1$ protein expression at different time-points in the experimental and control groups.

\begin{tabular}{lccccccr}
\hline Group & Day 1st & Day 3 & Day 7 & Day 14 & Natural death group & F & P-value \\
\hline Control & $115.63 \pm 14.38$ & $109.71 \pm 14.95$ & $152.86 \pm 16.74$ & $220.58 \pm 16.24$ & $185.68 \pm 16.63$ & 96.89 & $<0.001$ \\
Experimental & $53.33 \pm 4.93$ & $53.10 \pm 9.26$ & $8.12 \pm 1.25$ & $176.77 \pm 13.31$ & $129.10 \pm 14.10$ & & \\
$t$ & -4.10 & -3.22 & -8.62 & -2.09 & -2.60 & & \\
P-value & 0.01 & 0.01 & $<0.001$ & 0.05 & 0.02 & \\
\hline
\end{tabular}

and greater omentum. Surgical treatment often fails to eradicate these cells; therefore, patient survival rates remain low. Postoperative chemotherapy could destroy micrometastases and residual tumors, and it is considered an effective treatment for postoperative gastric cancer metastasis. However, due to the existence of the peritoneal serous membrane barrier, 

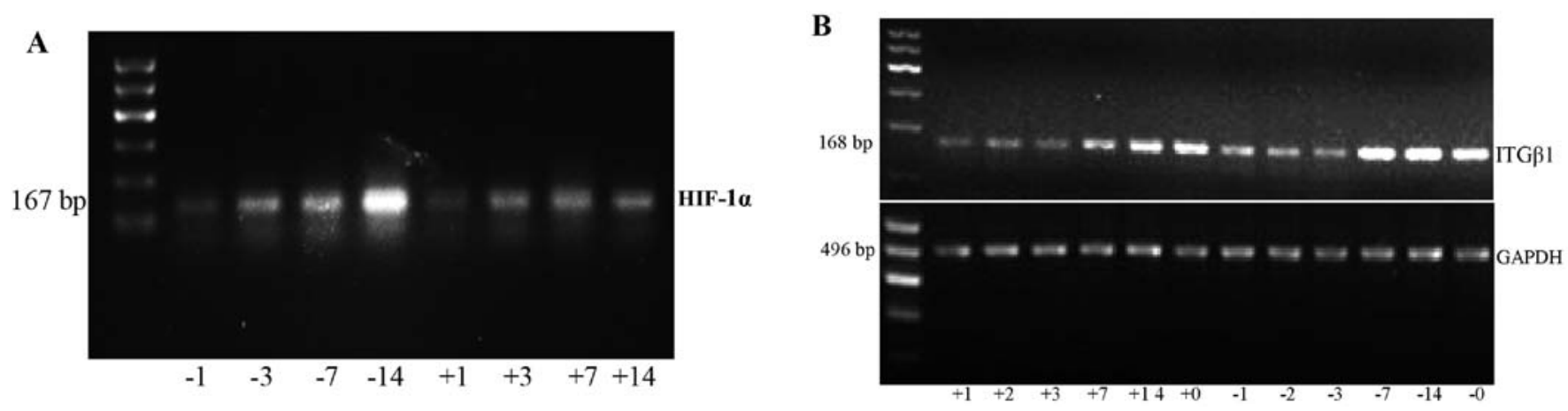

Figure 6. RT-PCR was used to detect the HIF-1 $\alpha$ (A) and ITG $\beta 1$ (B) mRNA expression in the experimental and control group in vivo. The labels $+1,+2,+3,+7$, +14 , and +0 indicate the experimental group at days $1,2,3,7$, and 14 and the natural death group, respectively. The labels $-1,-2,-3,-7,-14$, and -0 indicate the control group at days $1,2,3,7$, and 14 and the natural death group, respectively.

Table IV. HIF-1 $\alpha$ mRNA expression in the experimental and control groups at different times.

\begin{tabular}{lcccccr}
\hline Group & Day 1 & Day 3 & Day 7 & Day 14 & F & P-value \\
\hline Control group & $1.26 \pm 0.47$ & $1.7 \pm 0.34$ & $2.71 \pm 0.44$ & $3.5 \pm 0.41$ & 100.944 & $<0.001$ \\
Experimental group & $0.91 \pm 0.18$ & $1.4 \pm 0.22$ & $1.94 \pm 0.47$ & $2.8 \pm 0.46$ & 27.963 & $<0.001$ \\
$t$ & 2.752 & 2.664 & 4.705 & 10.968 & & \\
P-value & 0.016 & 0.015 & 0.001 & $<0.001$ & & \\
\hline
\end{tabular}

Table V. ITG $\beta 1$ mRNA expression in the experimental and control groups at different times.

\begin{tabular}{lcccccccr}
\hline Group (day) & Day 1 & Day 2 & Day 3 & Day 7 & Day 14 & Natural death & F & P-value \\
\hline Control group & $1.28 \pm 0.08$ & $1.3 \pm 0.05$ & $1.44 \pm 0.09$ & $2.5 \pm 0.02$ & $2.24 \pm 0.11$ & $2.59 \pm 0.11$ & 190.67 & 0.001 \\
Experimental group & $0.75 \pm 0.03$ & $0.7 \pm 0.03$ & $0.97 \pm 0.03$ & $1.3 \pm 0.04$ & $1.50 \pm 0.05$ & $1.83 \pm 0.03$ & & \\
$t$ & -6.43 & -7.90 & -5.33 & -26.12 & -4.66 & -18.98 & & \\
P-value & 0.003 & 0.001 & 0.006 & $<0.001$ & 0.01 & $<0.001$ & & \\
\hline
\end{tabular}

most of the intravenous chemotherapeutic drugs fail to reach the abdominal cavity, and drugs directly injected into the abdominal cavity more easily achieve effective concentrations. Common chemotherapeutic drugs have a small molecular weight and are absorbed easily in the abdominal cavity; therefore, their efficacy is reduced. The present study used DS, a type of macromolecular dextran derivative with a molecular weight of 500,000; this substance is absorbed slowly in the abdominal cavity and therefore has a high level of safety and few side effects. Guided by results that indicate that DS suppresses cell adhesion and cell cycle progression, both of which are essential for metastasis, DS could be used as an anti-metastatic drug (9). The present study used DS to prevent the growth of gastric cancer cells in a nude mouse celiac implantation model and administered the same amount of PBS or normal saline to a control group to study the possible mechanism by which DS inhibits the peritoneal metastasis of gastric cancer cells.

During tumor invasion of the surrounding tissue, which occurs in the process of metastasis, active cell mobility is an important factor. A lack of oxygen can improve tumor cell mobility and migration and is closely related to tumor metastasis (2). The oxygen level is a basic characteristic of the solid tumor microenvironment and can affect multiple stages of tumor development. When tumor growth reaches a particular stage, such that oxygen demand exceeds the supply or immature blood vessels within the tumor stroma rise and collapse, the local microenvironment enters a hypoxic condition. HIF-1 is primarily composed of HIF- $1 \alpha$ and HIF- $1 \beta$ subunits. HIF-1 $\alpha$ is the only oxygen-sensitive subunit and is primarily expressed in hypoxic cells (10). In a hypoxic microenvironment, cancer cells highly express HIF-1 $\alpha$ (3), which is the primary factor in the hypoxic state, and plays an active specific role in the hypoxic response. HIF-1 $\alpha$ is widely found in mammals and humans and can connect upstream and downstream signals in the process of oxygen activation. HIF-1 $\alpha$ allows cells to adapt to a hypoxic microenvironment and stimulates tumor growth and metastasis by promoting cell EMT, the regulation of angiogenesis (via VEGF) and increased cell adhesion (via ITG $\beta 1$ ) $(4,5)$. Therefore, ITG $\beta 1$ is important in the prevention and treatment of tumor recurrence along with HIF-1 $\alpha$. 
A
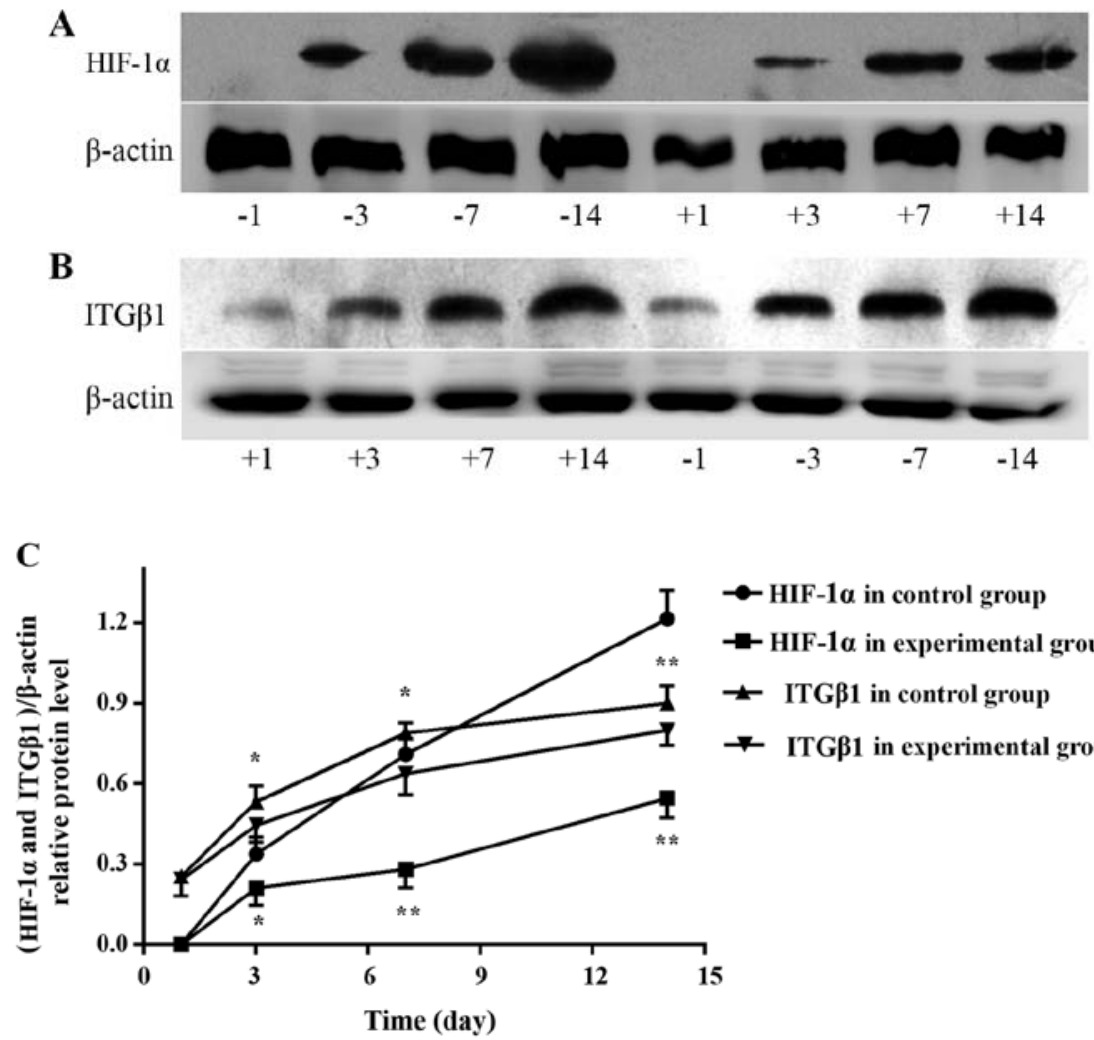

Figure 7. Electrophoresis banding of HIF-1 $1 \alpha$ (A) and ITG $\beta 1$ (B) protein in the experimental and control groups. (C) Line graph of the gray values of the relative HIF- $1 \alpha$ and ITG $\beta 1$ protein expression. The labels $+1,+3,+7$, and +14 indicate the experimental group at days $1,3,7$ and 14 , respectively, and the labels $-1,-3,-7$, and -14 indicate the control group at days 1,3,7 and 14, respectively. At days 3, 7 and 14, HIF-1 $\alpha$ and ITG $\beta 1$ expression levels were significantly lower in the experimental group than levels noted in the control group. ${ }^{*} \mathrm{P}<0.05$ or ${ }^{* *} \mathrm{P}<0.01$, difference between the experimental group and the control group.

Table VI. Correlation of HIF-1 $\alpha$ and ITG $\beta 1$ protein levels and drug and cell injection times in vivo.

\begin{tabular}{|c|c|c|c|c|c|}
\hline & & Injection time & $\begin{array}{c}\text { Tumor nodule } \\
\text { number }\end{array}$ & $\begin{array}{c}\text { HIF-1 } \alpha \text { protein } \\
\text { level }\end{array}$ & $\begin{array}{c}\text { ITG } \beta 1 \text { protein } \\
\text { level }\end{array}$ \\
\hline \multirow[t]{3}{*}{ Injection time } & Pearson's correlation & 1 & $0.908^{\mathrm{a}}$ & $0.847^{\mathrm{a}}$ & $0.932^{\mathrm{a}}$ \\
\hline & Significance (two-sided) & & 0.002 & 0.008 & 0.001 \\
\hline & $\mathrm{N}$ & 8 & 8 & 8 & 8 \\
\hline \multirow[t]{3}{*}{ Tumor nodule number } & Pearson's correlation & $0.908^{\mathrm{a}}$ & 1 & $0.991^{\mathrm{a}}$ & $0.941^{\mathrm{a}}$ \\
\hline & Significance (two-sided) & 0.002 & & 0.000 & 0.000 \\
\hline & $\mathrm{N}$ & 8 & 8 & 8 & 8 \\
\hline \multirow[t]{3}{*}{ HIF-1 $\alpha$ protein } & Pearson's correlation & $0.847^{\mathrm{a}}$ & $0.991^{\mathrm{a}}$ & 1 & $0.909^{\mathrm{a}}$ \\
\hline & Significance (two-sided) & 0.008 & 0.000 & & 0.002 \\
\hline & $\mathrm{N}$ & 8 & 8 & 8 & 8 \\
\hline \multirow[t]{3}{*}{ ITG $\beta 1$ protein } & Pearson's correlation & $0.932^{\mathrm{a}}$ & $0.941^{\mathrm{a}}$ & $0.909^{\mathrm{a}}$ & 1 \\
\hline & Significance (two-sided) & 0.001 & 0.000 & 0.002 & \\
\hline & $\mathrm{N}$ & 8 & 8 & 8 & 8 \\
\hline
\end{tabular}

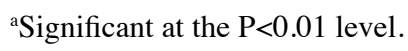

Here, we exposed gastric cancer BGC-823 cells to hypoxia to stimulate HIF-1 $\alpha$ expression and to observe whether HIF-1 $\alpha$ expression was affected by DS. We found that HIF- $1 \alpha$ mRNA and protein expression levels in the experimental and control groups were positively correlated with time in hypoxia and that DS reduced the expression of HIF-1 $\alpha$ in a hypoxic environment.
DS prevented cell adaptation to a hypoxic environment and reduced tumor growth and metastasis by inhibiting the expression of HIF- $1 \alpha$. This finding requires further experimental and clinical validation.

In addition, the increased expression of HIF-1 $\alpha$ can increase cell adhesion (through ITG $\beta 1$ ) as cells adapt to a hypoxic 
microenvironment, promoting tumor growth and metastasis. This process can occur through the transcriptional regulatory mechanism to promote ITG $\beta 1$ expression and function. Subsequent studies identified functional hypoxia-inducible activity, mediated by HIF, in the distal 5'-region of the ITGB1 promoter (11). Adherence plays an important role in the process of gastric cancer metastasis. Integrins are a family of adhesion factors, and ITG $\beta 1$ is an important $\beta$ subunit of the integrin family. ITG $\beta 1$ can combine with different $\alpha$ subunits to constitute important receptors in the extracellular matrix. Integrin plays a primary role in the occurrence and development of tumors: i) by mediating the adhesion of tumor cells and extracellular matrix, thereby promoting tumor cell invasion and metastasis. ii) The signals to cells from the extracellular matrix affect cell growth and differentiation. Abnormal integrin signaling promotes tumor cell growth and stimulates differentiation and distant metastasis (12). Research has shown that when tumor cells enter the circulation in a free state, the enhanced expression of ITG $\beta 1$ facilitates cancer cell adhesion, implantation and transfer $(12,13)$.

Research has shown that dysregulated ITG $\beta 1$ expression is related to cancer celiac implantation (7) and plays a key role in gastric cancer cell adherence to the peritoneum. The hTERT/MDM2-FOXO3a-ITGB1 pathway markedly contributes to gastric cancer invasion (14), suggesting that ITG $\beta 1$ may be a novel target for the prevention and treatment of gastric cancer metastasis. Other studies have suggested that an intraperitoneal injection of the ITG $\beta 1$ monoclonal antibody can significantly reduce adhesion, inhibit cancer cell peritoneal implants, and reduce the number of peritoneal implanting nodules (15). We observed ITG $\beta 1$ expression in gastric cancer cells and found that DS reduced ITG $\beta 1$ expression and inhibited gastric cancer cell adhesion, thus inhibiting gastric cancer metastasis.

The above in vitro experimental results indicated that DS can inhibit HIF-1 $\alpha$ and ITG $\beta 1$ expression in gastric cancer cells and cell adhesion. Because of the close relationship of HIF-1 $\alpha$ and ITG $\beta 1$ and their important roles in tumor cell metastasis, we analyzed the correlation between the two proteins. Both HIF- $1 \alpha$ and ITG $\beta 1$ expression were strongly related to intervention time, and HIF-1 $\alpha$ expression was positively correlated with ITG $\beta 1$ expression in both the experimental group and the control group. We hypothesized that HIF-1 $\alpha$ promotes tumor cell adaptation to a hypoxic microenvironment, thereby promoting tumor cell survival, growth and metastasis, by regulating the downstream factor ITG $\beta 1$.

Animal experiments have shown that DS can prevent B-16 melanoma cell growth in the greater omentum and peritoneal planting and can prolong the survival of mice with carcinoma of peritonitis (8). To further verify the impact of DS on gastric cancer celiac implantation, we used a series of nude mice that were administered a celiac injection of gastric cancer cells. The results showed that the number of tumor nodules markedly increased with the extension of the time since cancer cell injection. Nodules appear in the greater omentum first and most frequently, but tumor nodules can also be found in the liver, spleen, stomach wall and mesentery. However, this study focused on the greater omentum tumor nodules. At each time point, the tumor nodules on the greater omentum in the experimental group were significantly reduced in number and volume compared with those of the control group. The tumor nodule number was significantly and positively correlated with the time since injection. Immunohistochemistry, RT-PCR and western blot test results showed that the mRNA and protein expression levels of HIF- $1 \alpha$ and ITG $\beta 1$ in the experimental group were significantly lower than those of the control group. In addition, ITG $\beta 1$ expression was positively correlated with HIF-1 $\alpha$ expression, and the expression of both ITG $\beta 1$ and HIF-1 $\alpha$ was positively correlated with injection time and tumor nodule number. We hypothesized that DS likely acts through the inhibition of HIF- $1 \alpha$ expression, which reduced ITG $\beta 1$ expression and inhibited cancer cell adhesion. Thus, the local tumor hypoxia state cannot be improved in the presence of DS and the tumor growth is restrained, eventually reducing celiac implantation and gastric cancer metastasis.

In summary, the above results demonstrate that the expression of ITG $\beta 1$ is related to HIF-1 $\alpha$ expression and that DS can reduce the expression of HIF- $1 \alpha$ and ITG $\beta 1$ in gastric cancer cells and the greater omentum, effectively restraining the growth of nodules in the omenta of nude mice and, to some extent, may inhibit the celiac implantation and metastasis of gastric cancer cells. The mechanism may involve the inhibition of HIF- $1 \alpha$, which reduces ITG $\beta 1$ expression and inhibits cancer cell adhesion; thus, the local tumor hypoxic condition persists, thereby reducing tumor metastasis. This study may help the development of new effective drugs for the prevention and treatment of gastric cancer celiac metastasis.

\section{Acknowledgements}

An English Language Service from American Journal Experts was used to help prepare the manuscript. This study was supported by the Ningxia Natural Science Foundation (NZ1085), National Natural Science Foundation of China (no. 81460370) and Ningxia Natural Science Foundation (no. NZ13143), Ningxia Science and Technology Support Projects (2002310201).

\section{References}

1. Yamaguchi H, Kitayama J,Emoto S, Ishigami H, Ito T, Hanafusa N and Watanabe T: Cell-free and concentrated ascites reinfusion therapy (CART) for management of massive malignant ascites in gastric cancer patients with peritoneal metastasis treated with intravenous and intraperitoneal paclitaxel with oral S-1. Eur J Surg Oncol 41: 875-880, 2015.

2. Jing SW, Wang YD, Kuroda M, Su JW, Sun GG, Liu Q, Cheng YJ and Yang CR: HIF-1 $\alpha$ contributes to hypoxia-induced invasion and metastasis of esophageal carcinoma via inhibiting E-cadherin and promoting MMP-2 expression. Acta Med Okayama 66: 399-407, 2012.

3. Li H, Chen J, Zen W, Xu X, Xu Y, Chen Q and Yang T: Effect of hypoxia inducible factor-1 antisense oligonucleotide on liver cancer. Int J Clin Exp Med 8: 12650-12655, 2015.

4. Bao B, Wang Z, Ali S, Kong D, Li Y, Ahmad A, Banerjee S, Azmi AS, Miele L and Sarkar FH: Notch-1 induces epithelial-mesenchymal transition consistent with cancer stem cell phenotype in pancreatic cancer cells. Cancer Lett 307: 26-36, 2011.

5. Lee SH, Lee YJ and Han HJ: Role of hypoxia-induced fibronectin-integrin $\beta 1$ expression in embryonic stem cell proliferation and migration: Involvement of PI3K/Akt and FAK. J Cell Physiol 226: 484-493, 2011.

6. Goggins BJ, Chaney C, Radford-Smith GL, Horvat JC and Keely S: Hypoxia and integrin-mediated epithelial restitution during mucosal inflammation. Front Immunol 4: 272, 2013. 
7. Niu $\mathrm{G}$ and Chen $\mathrm{X}$ : Why integrin as a primary target for imaging and therapy. Theranostics 1: 30-47, 2011.

8. Hagiwara A, Sakakura C, Yamasaki J, Togawa T, Sonoyama Y, Fujiyama $J$ and Yamagishi H: Dextran sulfate inhibits injured abdominal wall-specific tumor implantation in mice. Anticancer Drugs 11: 873-877, 2000.

9. Takagi T, Sakakura C, Kin S, Nakase Y, Fukuda K, Shimomura K, Ito T, Fujiyama J, Yamasaki J, Tsujimoto H, et al: Dextran sulfate suppresses cell adhesion and cell cycle progression of melanoma cells. Anticancer Res 25: 895-902, 2005.

10. Kitajima Y and Miyazaki K: The critical impact of HIF-1a on gastric cancer biology. Cancers (Basel) 5: 15-26, 2013.

11. Keely S, Glover LE, MacManus CF, Campbell EL, Scully MM, Furuta GT and Colgan SP: Selective induction of integrin beta1 by hypoxia-inducible factor: Implications for wound healing. FASEB J 23: 1338-1346, 2009.

12. Yeh YC, Lin HH and Tang MJ: A tale of two collagen receptors, integrin $\beta 1$ and discoidin domain receptor 1 , in epithelial cell differentiation. Am J Physiol Cell Physiol 303: C1207-C1217, 2012.
13. Zhang $\mathrm{L}$ and Zou $\mathrm{W}$ : Inhibition of integrin $\beta 1$ decreases the malignancy of ovarian cancer cells and potentiates anticancer therapy via the FAK/STAT1 signaling pathway. Mol Med Rep 12: 7869-7876, 2015.

14. Hu C, Ni Z, Li BS, Yong X, Yang X, Zhang JW, Zhang D, Qin Y, Jie MM, Dong H, et al: hTERT promotes the invasion of gastric cancer cells by enhancing FOXO3a ubiquitination and subsequent ITGB1 upregulation. Gut: Sep 14, 2015 (Epub ahead of print). doi: 10.1136/gutjnl-2015-309322.

15. Lynch L, Vodyanik PI, Boettiger D and Guvakova MA Insulin-like growth factor I controls adhesion strength mediated by alpha5beta1 integrins in motile carcinoma cells. Mol Biol Cell 16: 51-63, 2005. 